


\title{
Sentido común y buen sentido en Deleuze
}

\section{Common sense and good sense in Deleuze}

\author{
Pablo Nicolás Pachilla \\ Universidad de Buenos Aires, Argentina \\ Université Paris 8, Francia
}

Resumen: Al ser el concepto de sentido común en Deleuze de primera importancia, resulta llamativo que no exista un trabajo interpretativo detallado al respecto. El presente artículo se propone subsanar dicha falta, ofreciendo un análisis riguroso de los diferentes sentidos y matices del concepto. Sostendremos que existen tres sentidos de "sentido común" en Deleuze: como Imagen del pensamiento, como concordia facultatum y como correlato del buen sentido. En los dos primeros sentidos, el sentido común se contrapone al empirismo trascendental deleuziano en tanto supone un pensamiento ya dado e impone una condición de concordancia en el ejercicio de las facultades; en el último, aporta la forma del objeto cualquiera que será cualificado por el buen sentido, que impone asimismo una determinación temporal escatológica y una determinación ontológica y espacial sedentaria.

Palabras clave: sentido común, buen sentido, Deleuze, concordancia, imagen del pensamiento.

Abstract: The concept of common sense is of vital importance in Deleuze's philosophy, but lacks nonetheless a detailed study. This paper aims to fill that gap, by offering a rigorous analysis of its different meanings and nuances. We will hold that there are three meanings of "common sense" in Deleuze: as the Image of thought, as concordia facultatum and as the correlate of good sense. In the first two cases, common sense 
conflicts with Deleuzean transcendental empiricism, insofar as it assumes a pre-given thought and imposes a condition of concordance between the faculties; in the latter, it provides the form of an object in general, which will be qualified by good sense. Good sense, in turn, imposes an eschatological temporal determination and a sedentary spatial and ontological determination to sense.

Keywords: Common sense, Good sense, Deleuze, Concordance, Image of thought.

Recibido: 1 de abril de 2018 Aceptado: 25 de septiembre de 2018

$\mathrm{E}$ n el presente artículo se analizará el concepto de sentido común tal como aparece en la filosofía de Gilles Deleuze, más allá de sus textos de carácter "monográfico". Para ello será necesario, en primer lugar, examinar el concepto deleuziano de Imagen del pensamiento, del cual tanto el sentido común como el buen sentido constituyen elementos indispensables. Nos centraremos principalmente en Différence et répétition y Logique du sens, pues son las obras en las que dichos conceptos son desarrollados con mayor precisión.

En el tercer capítulo de Diferencia y repetición, intitulado "La imagen del pensamiento", Deleuze despliega ocho postulados que caracterizan dicha imagen, también llamada "imagen dogmática", "imagen moral" o "imagen ortodoxa del pensamiento" (Deleuze, 1968: 172). ${ }^{1}$ Imagen del pensamiento quiere decir en este contexto: una noción no-cuestionada de lo que significa pensar. ${ }^{2}$ Más

${ }^{1}$ Todas las traducciones son de nuestra autoría salvo que se indique lo contrario. 2 En este sentido, Anne Sauvagnargues sostiene que "Deleuze retoma la iniciativa kantiana de una crítica del pensamiento, y le asigna a su vez el rol de examinar sus zonas de debilidades crónicas, esa ilusión trascendental a la que llama la imagen del pensamiento. El empirismo trascendental consiste en una clínica del pensamiento, que busca garantizar un empirismo purgado de las ilusiones de 
allá de los presupuestos objetivos que están presentes a la hora de comenzar a filosofar, Deleuze señala la existencia de presupuestos subjetivos. Por ejemplo, las nociones escolásticas de animalidad y racionalidad con las que Descartes elige no comprometerse, y por lo tanto evita definir al hombre como animal racional, constituyen un caso de presupuestos objetivos; las nociones de pensar y de ser implícitas en el camino cartesiano, no obstante, forman un ejemplo de presupuestos subjetivos. Lo que Deleuze hace en dicho capítulo es visibilizar los prejuicios sobre lo que significa pensar y, a partir de este cuestionamiento, esbozar una nueva imagen que, puesto que va a surgir del derrumbe de la imagen preexistente, llamará "pensamiento sin imagen". Esta expresión no precisa ser tomada al pie de la letra, y puede también pensarse en términos de una nueva imagen del pensamiento: lo importante en este contexto no es la palabra "imagen", sino el carácter presupuesto de los postulados que la caracterizan. En Pourparlers, Deleuze describe el concepto de imagen del pensamiento del siguiente modo:

Supongo que hay una imagen del pensamiento que varía mucho, que ha variado mucho en la historia. Por imagen del pensamiento no entiendo el método, sino algo más profundo, siempre presupuesto, un sistema de coordenadas, de dinamismos, de orientaciones: lo que significa pensar, y "orientarse en el pensamiento" (Deleuze, 1990: 202).

En esta línea, Deleuze y Guattari definirán la disciplina de la noología como "el estudio de las imágenes del pensamiento, y de su historicidad” (Deleuze/Guattari, 1980: 466). En el contexto de Diferencia y repetición, sin embargo, Deleuze reserva el término

la trascendencia, exponiendo los modos operatorios del pensamiento, que dan cuenta de su inventividad pero también de su conformismo" (Sauvagnargues, 2009: 9). 
“imagen del pensamiento" para referirse a una en particular, verbigracia, a la imagen dogmática. Más que identificar plenamente a ciertos filósofos con la Imagen del pensamiento, es preciso tomar este concepto como una figura que, si bien toma el rostro de incontables pensadores, no lo hace en todo momento, y en las grandes obras de la filosofía siempre se encuentran en mayor o menor medida momentos de ruptura o ambivalencia con respecto a dicha imagen. La imagen dogmática del pensamiento puede también caracterizarse como una imagen concordante, mientras que la propuesta deleuziana consiste en construir una imagen discordante del pensamiento.

Deleuze seńala ocho postulados que caracterizan a la Imagen del pensamiento. Éstos se refieren respectivamente a: 1) el pensamiento natural; 2) el sentido común y el buen sentido; 3) el reconocimiento; 4) la representación; 5) el error como contracara o peligro del pensamiento; 6) la designación (y no el sentido) como el lugar de la verdad; 7) el predominio de las soluciones por sobre los problemas, y 8) el predominio del saber por sobre el aprendizaje. El lugar del sentido común en la imagen del pensamiento, no obstante, es ubicuo: en la recapitulación final, Deleuze llama propiamente "postulado del ideal o del sentido común" (Deleuze, 1968: 216) al segundo de los postulados, pero en el transcurso del capítulo llama también "sentido común" al primer postulado (171), que identifica al mismo tiempo con la Imagen del pensamiento en tanto tal (172). Por otra parte, y en un sentido más restringido, indica que el segundo postulado se divide a su vez en sentido común, por un lado, y buen sentido, por otro. Hay entonces tres sentidos en juego en "sentido común": en primer lugar, es el segundo postulado, relativo a la concordancia entre las facultades; en segundo lugar, es una forma universal de identidad que funciona como correlato del buen sentido en tanto norma de repartición; por último, es la imagen del pensamiento en tanto tal. 
Sentido común e imagen del pensamiento son, en este último sentido, expresiones equivalentes. Exploraremos a continuación este tercer sentido global.

\section{Sentido común como imagen del pensamiento}

El primer postulado tiene un doble aspecto: la buena naturaleza del pensador y la naturaleza recta del pensamiento. El primer aspecto consiste en suponer que el pensamiento es el ejercicio natural de una facultad, es decir, que ya pensamos desde siempre y que lo hacemos naturalmente, mientras que el segundo indica que el pensamiento tiene una afinidad natural con lo verdadero. Si volvemos a la constatación evidente pero no por ello menos necesaria de que

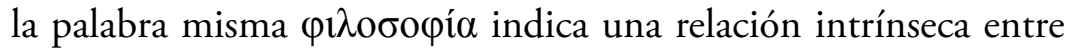
$\varphi \imath \lambda \varepsilon \tilde{i} v$ y $\sigma o \varphi i ́ \alpha$, se vuelve preciso concluir que la crítica deleuziana a la imagen del pensamiento es inherentemente una crítica a la filosofía en tanto tal. ¿Qué es, entonces, lo que hace Deleuze? Ante todo, criticar una tradición de pensamiento e intentar esbozar otro tipo de relación entre el deseo y la verdad.

En este sentido Deleuze se acerca a Heidegger, cuya filosofía de la diferencia es mencionada ya en el "Prefacio" como uno de los signos de la época. La fórmula heideggeriana "todavía no pensamos" en Was heißt denken? resume, para Deleuze, esta oposición a la cogitatio natura universalis. "El hombre puede pensar, en tanto tiene esa posibilidad. Sólo que esta posibilidad no nos garantiza que seamos capaces de realizarla." (Heidegger, 2002: 5) Hay que hacer advenir el pensamiento; es la misma idea que Deleuze rescatará de Artaud formulada en términos de la "genitalidad" del pensamiento como opuesta al innatismo (Deleuze, 1968: 192). Pero es precisamente esta inercia en la que ya siempre encontramos, tanto para Deleuze como para Heidegger, lo que más amerita ser pensado. "Lo que más da que pensar [Das Bedenklichste]", 
dice Heidegger, "es que todavía no pensamos; ni aun ahora que el estado del mundo da cada vez más que pensar [der Weltzustand fortgesetzt bedenklicher wird]" (Heidegger, 2002: 6).

El concepto heideggeriano de das Bedenklichste encuentra un equivalente en el concepto deleuziano de bêtise. Deleuze se pregunta, en este sentido: "si el pensamiento sólo piensa constreñido y forzado, si permanece estúpido en tanto que nada lo fuerza a pensar, ¿aquello que lo fuerza a pensar no es también la existencia de la necedad [bêtise], a saber, que él no piensa en tanto que nada lo fuerza?” (Deleuze, 1968: 353). Heidegger se expresaba en términos similares: "Lo que más da que pensar en nuestra preocupante época [Das Bedenklichste in unserer bedenklichen Zeit] es que todavía no pensamos" (Heidegger, 2002: 7). El término alemán Bedenklichste significa literalmente "preocupante", pero al contener la raíz - denk incluye la acepción de "lo que da que pensar". Heidegger lo define del siguiente modo: "das Bedenklichste [...] ist das, was uns zu denken gibt" (lo "más preocupante" es lo que nos da que pensar) (Heidegger, 2002: 7).

Si bien Deleuze reprochará a Heidegger su concepto de pre-

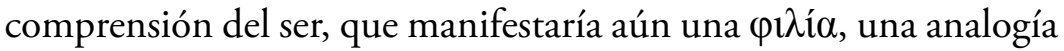
o una homología entre el pensamiento y lo que ha de ser pensado (Deleuze, 1968: 188), las afinidades con el pensador de la diferencia ontológica son profundas en lo que concierne a la pregunta “¿qué significa pensar?” Precisamente en el curso de 1951-1952 que lleva por título dicha pregunta, Heidegger distingue cuatro modos de realizarla:

La pregunta “¿qué significa pensar?” se deja preguntar de cuatro maneras. Ella pregunta:

1. ¿Qué es designado con la palabra pensar?

2. ¿Qué se entiende por pensar, es decir, qué entiende por eso la doctrina del pensar hasta ahora existente, la lógica? 
3. ¿Qué se requiere para que efectuemos el pensar correctamente?

4. ¿Qué es lo que nos encomienda [befiehlt] al pensar?

Nosotros sostenemos: la pregunta enumerada en cuarto lugar ha de ser preguntada en primer lugar (Heidegger, 2002: 127).

Deleuze parte de un mismo modo de preguntar, aunque sus respuestas varíen. Lo que nos encomienda al pensar es, para el filósofo francés, la intensidad dada en la sensibilidad. Se requiere primero de una afección, con la dimensión pasiva y contingente que ello reviste, para que el pensamiento nazca en nosotros. Recién en ese punto se puede desplegar correctamente la segunda pregunta enumerada por Heidegger, que corresponde a la imagen dogmática del pensamiento o Imagen del pensamiento. Y a partir de ahí se puede responder a la tercera pregunta, es decir, distinguir entre la imagen dogmática y la nueva imagen, o entre la Imagen del pensamiento y el pensamiento sin imagen. La primera pregunta, aunque insistente en todo el recorrido, será abordada por Deleuze en toda su dimensión sobre el final de su vida, constituyendo el problema de ¿Qué es la filosofía? (1991)

El punto de ruptura de Deleuze con Heidegger a este respecto se ubica en la direccionalidad de lo que da que pensar hacia el pensante. Dice el filósofo alemán: "El llamado proviene ya, en verdad, de ahí adonde se dirige el llamado. En el llamado está obrando un originario tender-hacia... [Auslangen nach...]" (Heidegger, 2002: 129). Este tender-hacia implica una afinidad natural del pensamiento con lo que ha de pensar, lo cual para Deleuze constituye un presupuesto de la imagen dogmática. Para él, por el contrario, el encuentro con la intensidad en tanto elemento que provoca el pensamiento es contingente y azaroso, y no existe en este sentido ningún "llamado" que presuponga direccionalidad desde lo llamante hacia lo llamado. Mientras que, según Heidegger, lo que da que pensar "nos confía el pensar en cuanto tal como destino de 
nuestra esencia [Wesensbestimmung]" (Heidegger, 2002: 130), no hay para Deleuze esencia ni destino alguno: la humanidad no está destinada a pensar.

Al señalar la conexión del recuerdo con la gratitud, Heidegger sostiene que "en el agradecimiento, el espíritu recuerda aquello en que permanece recogido en tanto que pertenece alli" (Heidegger, 2002: 150). Por el contrario, en la filosofía de Deleuze, el pertenecer no es nunca un lugar adonde volver, sino siempre un lugar de paso. El espíritu no pertenece a ningún lugar, puesto que toda territorialización es siempre un movimiento precedido por una desterritorialización, y a su vez pasible de ser sucedido por otra. Volver a la morada, a la patria, al hogar, como si allí hubiese algo más originario, más verdadero o más puro es un movimiento de re-territorialización que se cierra a otros devenires, y cuyas consecuencias políticas indeseables la historia nos revela claramente.

Volviendo a Diferencia y repetición, escribe Deleuze con respecto a la identificación del primer postulado con el sentido común:

La forma más general de la representación está entonces en el elemento de un sentido común como naturaleza recta y buena voluntad [...]. El presupuesto implícito de la filosofía se encuentra en el sentido común como cogitatio natura universalis, a partir del cual la filosofía puede tomar su punto de partida (Deleuze, 1968: 171).

Asimismo, puesto que los postulados no son proposiciones explícitas sino sobreentendidos pre-filosóficos, "el pensamiento conceptual filosófico tiene como presupuesto implícito una Imagen del pensamiento pre-filosófica y natural, tomada en préstamo al elemento puro del sentido común" (Deleuze, 1968: 172). Siempre que el punto de partida del filosofar sea esta imagen pre-filosófica, el punto de llegada no será más que una confirmación sofisticada del mismo. Es exactamente lo que el autor enfatizaba en La filosofía critica de Kant con esta cita algo modificada de la Crítica de 
la razón pura: "La más alta filosofía, en relación a los fines esenciales de la naturaleza humana, no puede conducir más lejos que la dirección acordada al sentido común" (Deleuze, 1963: 33). De lo que se trata entonces es de romper con ese punto de partida. ¿Cómo se empieza a pensar, entonces, si ni siquiera sabemos lo que significa pensar? La apuesta de Deleuze reside en la experimentación. De allí la fórmula empirismo trascendental, que utiliza para describir su filosofía:

Empirismo trascendental no quiere decir nada efectivamente si no se precisan las condiciones. El "campo" trascendental no debe ser calcado de lo empírico, como lo hace Kant: debe por su lado ser explorado en tanto tal, por ende "experimentado" (pero [se trata] de un tipo de experiencia muy particular). Es este tipo de experiencia el que permite descubrir las multiplicidades, pero también el ejercicio del pensamiento [...]. Puesto que creo que, además de las multiplicidades, lo más importante para mí ha sido la imagen del pensamiento tal como he intentado analizarla en Diferencia y repetición, en Proust, y por todas partes (Deleuze, 2003: 339).

El pensamiento es algo que sólo puede experimentarse, algo que sucede inesperadamente, y nunca lo que creíamos saber que era; es por ello que Artaud, con su noción de genitalidad, plantea según Deleuze "el principio de un empirismo trascendental" (Deleuze, 1968: 192). Como señala Christian Kerslake aludiendo a los conceptos kantianos de Umfang y Schranken, "el rango y las limitaciones de las facultades se descubren en su propio ejercicio, antes que ser pre-dados" (Kerslake, 2009: 81). Esto implica asimismo, como indica Marc Rölli, "la inversión necesaria de las relaciones entre lo trascendental y lo empírico" (Rölli, 2003: 1). El sentido común, en este contexto, implica por el contrario todo aquello que ya sabíamos de antemano que significaba pensar: la no-filosofía como fundamento de la filosofía. 
Desde un punto de vista filológico, es plausible afirmar que Deleuze toma el término "empirismo trascendental" del Traité de métaphysique de Jean Wahl, donde se lee:

Cuando Kant dijo que el ser es esencialmente posición, proveyó sin duda el punto de partida para la filosofía positiva de Schelling y para lo que podríamos llamar un empirismo superior. Hay en efecto un empirismo trascendental, precisamente el empirismo de Schelling, que busca las condiciones, no digamos de la posibilidad, sino de la realidad de la experiencia (Wahl, 1953: 324).

No sólo fue Deleuze alumno de Wahl en la Sorbonne, sino que el libro citado era utilizado como manual en sus clases. ${ }^{3}$ Deleuze se refiere en Diferencia y repetición al libro de Wahl, Les Philosophies pluralistes d'Angleterre et d'Amérique, dedicado al pragmatismo anglo-norteamericano aunque centrado primordialmente en la filosofía de William James. Allí, Deleuze escribe que "[t]oda la obra de Jean Wahl es una profunda meditación sobre la diferencia; sobre las posibilidades de expresar su naturaleza poética, libre y salvaje; sobre la irreductibilidad de la diferencia a lo negativo; sobre las re-

\footnotetext{
${ }^{3}$ En la bibliografía sumaria al final de DR figura el nombre de Wahl pero no se indica ningún texto en particular, sino un vago "passim" (Deleuze, 1968: 402); es decir, su obra entera. Ello refuerza nuestra hipótesis de que Deleuze tenía presente el trabajo de Wahl más allá de Les Philosophies pluralistes.. Por otra parte, en sus Diálogos con Claire Parnet, Deleuze dice que Wahl era "el filósofo más importante en Francia”, con excepción de Sartre (Deleuze/Parnet, 1996: 72). Tanto Gregory Flaxman como Stéphane Madelrieux se ocupan de la importancia de Wahl en el pensamiento de Deleuze, pero refiriendo siempre de manera exclusiva a Les Philosophies pluralistes... (Cf. Madelrieux, 2015: 89-104). El término "empirismo trascendental" había sido utilizado previamente, aunque en contextos menos cercanos a Deleuze. Como señala Rölli, el término es utilizado por Ludwig Landgrebe para referirse a Husserl, quien habría planteado su propia filosofía como una culminación tanto de la filosofía trascendental kantiana como del empirismo humeano (Cf. Rölli, 2003: 11).
} 
laciones no-hegelianas entre la afirmación y la negación" (Deleuze, 1968: 81), y se sirve de un poeta citado por Wahl para describir "la profesión de fe del empirismo trascendental como verdadera estética" (Deleuze, 1968: 80). ${ }^{4}$ Con ello, la tarea de exploración del campo trascendental, esa "pura corriente de conciencia a-subjetiva, conciencia pre-reflexiva impersonal, duración cualitativa de la conciencia sin yo" (Deleuze, 2003: 359), es puesta primordialmente en el campo de experimentación sensible. Esta exploración sólo puede abrirse, empero, a partir de una ruptura con las condiciones de concordancia impuestas por el sentido común y la imagen dogmática.

\section{Sentido común como concordia facultatum}

El sentido común en tanto segundo postulado de la imagen del pensamiento se refiere a un determinado modelo de relación entre las distintas facultades subjetivas. El modelo en cuestión es el del reconocimiento, por lo cual el segundo y el tercer postulado son

\footnotetext{
${ }^{4}$ Esta profesión de fe es atribuida al filósofo y poeta neoyorkino Benjamin Paul Blood (1822-1919), de quien Deleuze cita el siguiente fragmento: "Nature is contingent, excessive and mystical essentially. [...] We have realized the highest divine thought of itself, and there is in it as much of wonder as of certainty. [...] Not unfortunately the universe is wild - game flavoured as a hawk's wing. Nature is miracle all. She knows no laws; the same returns not, save to bring the different. The slow round of the engraver's lathe gains but the breadth of a hair, but the difference is distributed back over the whole curve, never an instant true - ever not quite" (Deleuze, 1994: 57). La traducción francesa utilizada por Deleuze es la del propio Wahl. Citamos aquí el original inglés, colocado en el lugar de la traducción francesa de Wahl en la edición en lengua inglesa de Différence et répétition. Deleuze remite en nota a pie de página no al propio Blood, quien publicara el mentado poema en 1874, ni a William James, quien lo cita en su ensayo "A Pluralistic Mystic: Benjamin Paul Blood" (1910), sino a la obra de Wahl. Como dice Flaxman, es entonces Wahl quien "expresa la profesión de fe del empirismo trascendental como verdadera estética” (Flaxman, 2015: 58).
} 
interdependientes. "El reconocimiento se define por el ejercicio concordante [concordant] de todas las facultades sobre un objeto que se supone el mismo: es el mismo objeto el que puede ser visto, tocado, recordado, imaginado, concebido..." (Deleuze, 1968: 174). Los ejemplos paradigmáticos son la cera cartesiana y la triple síntesis kantiana; es claro, en efecto, que Deleuze está pensando aquí en Descartes y Kant. Teniendo en cuenta su estudio sobre el segundo, Deleuze precisa que esto no implica que cada facultad no aporte su dato específico o que no tenga su particularidad: lo sensible no es el mismo tipo de flujo que lo memorable ni que lo concebible; cada facultad difiere en naturaleza. Sin embargo, cada una da su aporte específico en función de una colaboración que culmina en el reconocimiento de un objeto: "un objeto es reconocido cuando una facultad lo mienta [vise] como idéntico al de otra, o más bien cuando todas las facultades conjuntamente remiten su dato y se remiten ellas mismas a una forma de identidad del objeto" (Deleuze, 1968: 174). Hay una condición de concordancia de los flujos, por la cual todos ellos se dirigen hacia un mismo objeto. Es importante señalar que este objeto, por su parte, no corresponde a ninguno de los flujos en particular; si lo real consiste en flujos, el objeto, en sí mismo, no es nada. Es tan sólo el punto de convergencia ideal de los flujos.

En este sentido, leemos en los apuntes del curso de hypokhâgne dado por Deleuze en 1956-1957:

Los fenómenos son lo que aparece. Conocer, ¿es sólo aprehender lo que aparece? No exactamente. Lo que aparece es un flujo de cualidades sensibles. Conocer es hacer de esas cualidades la cualificación de algo. Kant: el objeto = x que es una función del conocimiento (Deleuze, 1956-1957). ${ }^{5}$

5 "Qu'est-ce que fonder?" Curso de hypokhâgne, Lycée Louis le Grand (19561957). Notas manuscritas disponibles en: webdeleuze.com. No hay paginación. 
Este "flujo de cualidades sensibles" es, para tomar la terminología de Prolegómenos, suficiente para realizar juicios de percepción; pero para realizar juicios de experiencia se necesita además "hacer de esas cualidades la cualificación de algo", viz., del objeto trascendental. ${ }^{6}$ Para tener conocimiento, debo poner la forma del objeto como centro vacío en el cual converjan esos flujos.

Falta todavía un elemento, sin embargo, puesto que la identidad objetiva en la que convergen las facultades no se sostiene por sí misma, sino que es necesariamente correlativa de la identidad subjetiva. Este es para Deleuze el principio del Cogito, y el Cogito como principio: "la forma de identidad del objeto reclama, para el filósofo, un fundamento en la unidad de un sujeto pensante del cual todas las otras facultades deben ser los modos" (Deleuze, 1968: 174). El pensamiento, en esta imagen del pensamiento, es aquello que unifica y abarca todas las facultades, y en este sentido trasciende la diferencia de naturaleza entre ellas, así como la inmanencia de sus flujos. "Se supone que el pensamiento es naturalmente recto porque no es una facultad como las otras, sino que, remitido a un sujeto, es la unidad de todas las otras facultades, que son solamente sus modos, y que él orienta a la forma de lo Mismo en el modelo del reconocimiento.” (Deleuze, 1968: 175) El pensamiento que surja de la crítica de esta imagen deberá entonces ser un flujo independiente, y no la mera forma de la unidad del resto de los flujos. Este sentido de "pensamiento" sólo puede surgir entre los intersticios de los distintos flujos, y por eso sólo puede nacer a partir del derrumbe de la imagen concordante o del sentido común. Ello explica que no exista de antemano, sino que requiera de

Se trata de los apuntes tomados por un estudiante, de nombre Pierre Lefebvre. ${ }^{6}$ Sobre la distinción kantiana entre juicios de percepción y juicios de experiencia, $c f$. el clásico, sintético y brillante Dotti (1986, pp. 239-242). 
un difícil y trabajoso parto en el cual la destrucción es tan necesaria como la construcción.

Esa unidad del sujeto pensante es para Kant la apercepción trascendental, el yo pienso que debe poder acompañar todas mis representaciones. " "En Kant como en Descartes, es la identidad del Yo $[M o i]$ en el Yo [Je] pienso lo que funda la concordancia [concordance] de todas las facultades, o su acuerdo [accord] en la forma de un objeto que se supone el Mismo" (Deleuze, 1968: 174). Nuevamente, que este Yo sea idéntico para cada una de "mis" representaciones es un postulado del que Deleuze quiere deshacerse. Desde luego, dicha identidad permitiría explicar una cantidad de fenómenos: que "yo" pueda recordar mis propias vivencias pero no las de otros, etc. Deleuze va a intentar dar cuenta de estos fenómenos por una vía empirista en el segundo capítulo de Diferencia y repetición mediante la primera síntesis del tiempo: hay múltiples yoes que se sintetizan progresivamente a través del hábito (Cf. Deleuze, 1968: 96-108).

\section{Sentido común y buen sentido}

Nos resta aún por ver el sentido más específico que da Deleuze a "sentido común" en el tercer capítulo de Diferencia y repetición así como también en Lógica del sentido, a saber, como correlato del buen sentido. Puesto que nunca nos encontramos con "el objeto

7 "El Yo pienso debe poder acompañar a todas mis representaciones; pues de otro modo, sería representado en mí algo que no podría ser pensado, lo que viene a significar, o bien que la representación sería imposible, o que, al menos, no sería nada para mí" (Kant, 2009: 202) (AA III, 108.19-22, B 132). Por eso, precisamente, sería un problema que hubiera flujos sensibles que no pudieran ser representados (pensados por mí): es allí donde radica la problematicidad de los pasajes kantianos referidos a una experiencia sin reconocimiento (Erscheinung en contraposición a Phänomen, juicio de percepción en contraste con juicio de experiencia, etc.). 
cualquiera" en tanto tal, sino siempre con un cierto y determinado objeto, debe haber una instancia que complemente al sentido común en tanto que proveedora de la forma de la objetividad como reflejo de la identidad subjetiva:

si el sentido común es la norma de identidad, desde el punto de vista del Yo [Moi] puro y de la forma de objeto cualquiera que le corresponde, el buen sentido es la norma de repartición [partage], desde el punto de vista de los yoes [moi] empíricos y de los objetos cualificados como tal o cual (es por lo cual se estima universalmente repartido). Es el buen sentido el que determina el aporte de las facultades en cada caso, cuando el sentido común aporta la forma de lo Mismo (Deleuze, 1968: 175).

En su curso de 1956-1957, asimismo, Deleuze no hablaba de sentido común, pero sí de buen sentido. Si bien todavía no había forjado la distinción entre distribución sedentaria y distribución nómade, ya allí el buen sentido es definido en términos distributivos, con expresiones como "la regla esencial del buen sentido es la repartición" o "[e]l buen sentido reparte la verdad en partes" (Deleuze, 1956-1957). Tres filósofos son invocados por Deleuze contra el buen sentido: Sócrates, Hegel y Marx. En cuanto al primero, Deleuze subraya que la doxa, la opinión o el buen sentido constituyen el enemigo contra el cual se erigió, y que es esta enemistad la que le costó la vida. De ese modo Deleuze construye allí una enemistad esencial entre la filosofía y el buen sentido que tenderá a abandonar con sus lecturas de Nietzsche: "[e]l buen sentido es el blanco de la filosofía” (Deleuze, 1956-1957). Esta concepción de la filosofía cambiará radicalmente a partir de Proust et les signes (1964), aunque seguirá siendo la concepción de la filosofía defendida por el autor. En cuanto a Hegel, Deleuze recuerda la oposición entre buen sentido y filosofía planteada en el Differenzschrift, donde la doxa o buen sentido es célebremente definida como el 
sentimiento de lo absoluto unido a la verdad parcial. ${ }^{8}$ Cabe aclarar que nada quedará de esta imagen positiva de Sócrates y de Hegel en el Deleuze posterior: ambos se convertirán, por el contrario, en representantes del sentido común devenido en filosofía. En cuanto a Marx, Deleuze trae a colación su crítica a Proudhon en Miseria de la filosofía, donde el mutualista francés es tratado de pequeñoburgués por creer que la dialéctica se trata de repartir: "por una parte, por otra parte". ${ }^{9}$ No es el único comentario deleuziano ligando el buen sentido a una determinada extracción social: "quizás

${ }^{8}$ Si bien el título del apartado en cuestión es "Relación de la especulación con el entendimiento común humano [gesunden Menschenverstand]", Hegel utiliza allí indiferentemente "sano entendimiento humano", "entendimiento común humano" y "entendimiento común"; en ningún caso utiliza, empero, la expresión Gemeinsinn (sentido común). "Lo que el llamado sano entendimiento humano [gesunde Menschenverstand] reconoce como racional también son singularidades extraídas de lo absoluto e introducidas en la conciencia; puntos luminosos que, aislados, se elevan de la noche de la totalidad. Con ellos, los hombres se orientan razonablemente en la vida; para ellos son puntos de vista correctos, de los que parten y a los que vuelven. Pero en realidad el hombre sólo tiene esta confianza en la verdad de tales singularidades porque la acompańa -en forma de un sentimiento- lo absoluto, y únicamente esto les da significado a las singularidades. En cuanto que estas verdades del entendimiento común humano [gemeinen Menschenverstandes] se toman para sí, en tanto que se aíslan, de un modo meramente intelectivo, como conocimientos en general, aparecen como desviadas y como medias verdades" (Hegel, 1968: 20). Juan Antonio Rodríguez Tous traduce gemeiner Menschenverstand por "sentido común" y gesunder Menschenverstand a veces por "sano sentido común" y a veces simplemente por "sentido común" (Cf. Hegel, 1989: 21-25). David Zapero Maier, por su parte, traduce ambas expresiones por "sentido común" (Cf. Hegel, 2012: 47-50).

9 "Para él, para Proudhon, cada categoría económica tiene dos lados, uno bueno y otro malo. Considera las categorías como el pequeñoburgués considera a las grandes figuras históricas: Napoleón es un gran hombre; ha hecho mucho bien, pero también ha hecho mucho mal. El lado bueno y el lado malo, la ventaja y el inconveniente, tomados en conjunto, forman según Proudhon la contradicción inherente a cada categoría económica. Problema a resolver: Conservar el lado bueno, eliminando el malo" (Marx, 1987: 69). Para Marx, Proudhon aplica una 
la filosofía encuentra su origen en la existencia misma de su enemigo, las clases medias" (Deleuze, 1956-1957). Las clases medias o la pequeña burguesía parecen en efecto caracterizarse para Deleuze por mediar, conciliar, repartir; es decir, por realizar la tarea del buen sentido.

En Diferencia y repetición y Lógica del sentido, esta concepción del buen sentido adquiere un sentido más técnico, pero mantiene el color otorgado en “¿Qué es fundar?” La distinción entre sentido común y buen sentido le permite a Deleuze identificar dos agentes complementarios de la representación, relativos a lo general y lo particular respectivamente. La objetividad (la forma del objeto) y la identidad subjetiva serían universales abstractos si no estuvieran conectados con la particularidad de lo empírico. Es por ello que el sentido común como forma necesita del buen sentido como materia: de este modo, lo que sería una mera abstracción si estuviese desligado de la experiencia, se convierte en un verdadero dispositivo de objetivación y sujeción al encabalgarse sobre los flujos de las facultades. El sentido común aporta la forma del objeto cualquiera, y el buen sentido su cualificación. El buen sentido es la instancia que se encarga de determinar qué de los flujos va a pasar y qué no va a pasar en un determinado ejercicio concordante de las facultades.

Así como el código social determina qué de los flujos pasa y qué no, el buen sentido como código subjetivo determina qué de lo sensible, de lo imaginable o de lo memorable pasará y qué no. "El código social quiere decir que algo del flujo debe pasar, correr; algo no debe pasar; y en tercer lugar, algo debe hacer pasar o bloquear." (Deleuze, 2010: 25) El buen sentido es esta tercera instancia. Lo que no pase, Deleuze va a pensarlo como el inconsciente, y es en este sentido que hay un inconsciente de la percepción, de la imaginación, etc.; desde el punto de vista de la imagen del pensamiento,

dialéctica hegeliana mal entendida. "A su juicio, el movimiento dialéctico es la distinción dogmática de lo bueno y de lo malo" (Marx, 1987: 70). 
sólo se puede hablar del inconsciente en general de manera negativa, exactamente como Kant se refiere a la cosa en sí, es decir, para mentar lo inefable que queda por fuera de la representación.

En la serie "Sobre la paradoja" de Lógica del sentido, Deleuze elabora la idea de una correlación entre la función subjetiva y la función objetiva del sentido común:

En el "sentido común", "sentido" no se dice ya de una dirección, sino de un órgano. Se lo llama común porque es un órgano, una función, una facultad de identificación, que remite una diversidad cualquiera a la forma de lo Mismo. El sentido común identifica, reconoce, así como el buen sentido prevé. Subjetivamente, el sentido común subsume facultades diversas del alma u órganos diferenciados del cuerpo, y los remite a una unidad capaz de decir Yo [Moi]: es un sólo y mismo yo [moi] el que percibe, imagina, recuerda, sabe, etc.; y el que respira, que duerme, que camina, que come... El lenguaje no parece posible por fuera de un sujeto tal, que se expresa o se manifiesta en él, y que dice lo que hace. Objetivamente, el sentido común subsume la diversidad dada y la remite a la unidad de una forma particular de objeto o de una forma individualizada de mundo: es el mismo objeto el que veo, huelo, pruebo, toco, el mismo que percibo, que imagino y que recuerdo... y es en el mismo mundo que respiro, que camino, que estoy despierto o que duermo, yendo de un objeto al otro de acuerdo con las leyes de un sistema determinado. Nuevamente, el lenguaje no parece posible por fuera de estas identidades que designa (Deleuze, 1969: 95-96).

La primera frase se explica por el hecho de que Deleuze viene de subrayar, como veremos en el apartado siguiente, que la palabra "sentido" en la fórmula "buen sentido" significa "dirección". Al sostener que en "sentido común", la palabra "sentido" significa ahora "órgano", Deleuze está admitiendo que hay una equivocidad en el significado de "sentido" entre sus conceptos de buen sentido 
y de sentido común. No es necesario recurrir a la vindicación deleuziana de la univocidad (del ser) para ver que esto podría representar un problema en esta construcción conceptual. Aceptar este equívoco intencional de un término tan central como "sentido" es algo que uno bien podría no estar dispuesto a hacer. Deleuze parece tener la convicción, no obstante, de que el constructo teórico "sentido común/buen sentido" tiene consistencia independientemente de dicha equivocidad. No podemos dejar de plantear la inquietante duda, sin embargo, respecto de si el tipo de unidad existente entre los dos significados de "sentido" no es un tipo de unidad analógica. Ello no necesariamente haría caer la relación de complementariedad entre estos dos conceptos, aunque debilitaría parcialmente la tesis de que se trata de un mismo dispositivo, puesto que la comunidad y la bondad ya no serían predicadas de lo mismo en "sentido común" que en "buen sentido". Creemos que la tesis se mantiene en pie siempre que Deleuze pueda mostrar que la buena dirección del sentido es el correlato necesario de un órgano identificatorio.

La referencia a las Meditaciones metafísicas en el pasaje citado, por su parte, no deja de aplicarse al criticismo kantiano. Por más que neguemos, con Kant, la posibilidad de hacer del sujeto una substancia o una cosa, sigue siendo necesario que sea una misma instancia la "que duda, que entiende, que afirma, que niega, que quiere, que no quiere, que imagina también, y que siente" (Descartes, 1997: 25). Lo que cambia en Deleuze con respecto a Kant es para qué es necesaria esa identidad subjetiva: mientras que para Kant es condición de posibilidad de la experiencia, para Deleuze es condición de posibilidad del lenguaje. Esta diferencia es importante, siempre que convengamos que puede haber experiencia sin lenguaje o, por lo menos, que hay capas pre-lingüísticas de la experiencia. Independientemente de ello, lo que enfatiza Deleuze es que sin esta identidad producida por el sentido común, no habría 
Yo unitario sino dispersión. Pero el sentido común no sólo identifica los flujos de la experiencia en un sujeto supuesto idéntico; en el otro extremo, los identifica asimismo en un objeto. Si los flujos de las facultades son líneas paralelas en tanto que difieren en naturaleza, el sentido común es un dispositivo de curvatura de los flujos, que los hace converger en las dos puntas. Sin embargo, puesto que hay diversidad de objetos, la unidad también se perdería si no hubiera un horizonte o marco que los abarcara a todos, y que permitiera que el sujeto se relacionara con distintos objetos sin perderse en el camino: de allí la necesidad de la Idea de mundo en Kant y del dispositivo-mundo en Deleuze ("es en el mismo mundo que respiro, que camino, que estoy despierto o que duermo, yendo de un objeto al otro de acuerdo con las leyes de un sistema determinado").

Un tercer punto a subrayar es la asignación de las actividades respectivas: la función propia del sentido común es la identificación o el reconocimiento, mientras que la función propia del buen sentido -como quedará claro en el próximo apartado- es la previsión. Su complementariedad es necesaria para el sistema de la representación tanto subjetiva como objetivamente: la identidad del Yo no subsistiría sin la identidad del objeto, y viceversa. Esta complementariedad entre sentido común y buen sentido se ve claramente en el siguiente pasaje:

El buen sentido no podría asignar ningún comienzo y ningún fin, ninguna dirección, no podría distribuir ninguna diversidad, si no se sobrepasara hacia una instancia capaz de remitir ese diverso a la forma de identidad de un sujeto, a la forma de permanencia de un objeto o de un mundo, que se suponen estar presentes desde el comienzo hasta el fin. Inversamente, esta forma de identidad en el sentido común permanecería vacía si no se sobrepasara hacia una instancia capaz de determinarla para tal o cual diversidad que comienza aquí, termina allá, y que se supone que dura todo el 
tiempo necesario para la igualación de sus partes. Es preciso que la cualidad sea a la vez detenida y medida, atribuida e identificada. Es en esta complementariedad del buen sentido y del sentido común que se anuda la alianza del yo [moi], del mundo y de DiosDios como término último de las direcciones y principio supremo de las identidades (Deleuze, 1969: 96).

Recordemos lo que decía Deleuze en su curso de hypokhâgne: "[1] o que aparece es un flujo de cualidades sensibles" (Deleuze, 19561957). Ese flujo debe ser detenido siempre que se pretenda conocer un objeto. La función sensible del buen sentido es precisamente realizar esa detención. En la serie "De la proposición", Deleuze escribe: "Del verde como color sensible o cualidad, distinguimos el 'verdear' como color noemático o atributo. ¿El árbol verdea, no es finalmente el sentido del color del árbol [...]?" (Deleuze, 1969: 33) Las cualidades, en efecto, pueden ser tomadas en su sentido independientemente de las riendas de lo bueno y lo común que se montan sobre ellas y las desnaturalizan. Pero ello implica la imposibilidad del conocimiento, que necesita identificar o reconocer objetos. Por ese motivo, el medio del buen sentido para detener el flujo y hacer posible el conocimiento es hacer que esas cualidades no sean aprehendidas en su inmanencia sin más, sino en su inmanencia a un objeto. Ese es el procedimiento de la cualificación: "[c] onocer es hacer de esas cualidades la cualificación de algo" (Deleuze, 1956-1957). De allí que Deleuze reproche a Platón su interpretación del devenir cualitativo en términos de contrariedad, puesto que la contrariedad en tanto que figura de la oposición resulta también en una detención del devenir-loco.

El descubrimiento platónico de este fenómeno es para Deleuze de primera importancia, dado que constituye, también para el propio Deleuze, el punto de partida del pensamiento en tanto aquello que nos fuerza a pensar. 
Mientras que el dedo no es nunca sino un dedo, lo duro no es nunca duro sin ser también blando, puesto que es inseparable de un devenir o de una relación que pone en él lo contrario [...]. Es entonces la coexistencia de contrarios, la coexistencia del más y del menos en un devenir cualitativo ilimitado, lo que constituye el signo o el punto de partida de lo que nos fuerza a pensar. El reconocimiento, al contrario, mide y limita la cualidad remitiéndola a algo, [y] detiene así el devenir-loco (Deleuze, 1968: 184).

Sin embargo, pensar este devenir a partir de la contrariedad como figura de la oposición indica para Deleuze que Platón confunde el ser de lo sensible (intensidad de la dureza) con el simple ser sensible o cualitativo (ser duro en oposición a ser blando). No reconocer es condición de posibilidad pero no condición suficiente para pensar: es necesario asimismo evitar atrapar el devenir en las formas de lo negativo, puesto que de ese modo se reintroduce subrepticiamente la forma de la identidad -en el caso de Platón, a través de las Ideas como pasibles de ser reconocidas, aunque no sea bajo la forma cartesiana del innatismo sino bajo la forma de la reminiscencia-. El innatismo y la reminiscencia como formas del reconocimiento señalan dos modelos distintos de comprender una temporalidad no-empírica que condiciona la experiencia: el primero, como negación del problema a través de la remisión a Dios, que es autor tanto de las ideas innatas a partir de las cuales reconocemos como de la creación continua del mundo en el que usamos esas ideas para reconocer; el segundo, como un tránsito por un pasado y un olvido míticos, aunque ambos concebidos a partir del presente en el cual los reconocemos, y por tanto bajo la figura empírica de antiguos presentes. En última instancia, es entonces la identidad de la Idea reconocida lo que lleva a Platón a pensar el devenir cualitativo ilimitado en términos de contrariedad, ya sea como mezcla entre dos identidades o entre una identidad y una instancia de carencia. 
Volviendo al pasaje de Lógica del sentido citado previamente, el final alude a la tripartición wollfiana tomada por Kant de la metaphysica specialis en psicología racional, cosmología racional y teología racional, tripartición que dará origen a las tres Ideas kantianas: Alma, Mundo y Dios. Deleuze coincide entonces con Kant en que las tres son necesarias; el punto, desde luego, es para qué. Nuevamente, mientras que en Kant son condiciones de posibilidad de la experiencia, en Deleuze son condiciones de posibilidad de la representación. Todo el punto de la filosofía deleuziana, no obstante, es mostrar que hay un tipo de experiencia no-representativa. En cuanto al "tiempo necesario para la igualación de sus partes", es preciso detenerse un momento en las nociones allí implicadas.

\section{El buen sentido como determinación temporal del sentido}

El buen sentido impone según Deleuze una estricta determinación temporal al sentido: la direccionalidad que va del pasado al futuro, identificando el primero con la diferencia y el segundo con la indiferencia. Leemos en la serie "Sobre la paradoja":

Ahora bien, el buen sentido se dice de una dirección: es sentido único, expresa la exigencia de un orden según el cual hay que elegir una dirección y atenerse a ella. Esta dirección es fácilmente determinada como la que va de lo más diferenciado a lo menos diferenciado, de la parte de las cosas a la parte del fuego. De acuerdo con ella se orienta la flecha del tiempo, puesto que lo más diferenciado aparece necesariamente como pasado en tanto que define el origen de un sistema individual, y lo menos diferenciado [aparece] como futuro y como fin. Este orden del tiempo, del pasado al futuro, es entonces instaurado por relación al presente, es decir, en relación con una fase determinada del tiempo elegida en el sistema individual considerado. El buen sentido se da así la con- 
dición bajo la cual cumple su función, que es esencialmente prever: es claro que la previsión sería imposible en la otra dirección, si se fuera de lo menos diferenciado a lo más diferenciado, por ejemplo, si temperaturas primero indiscernibles se fueran diferenciando. Es por ello que el buen sentido se ha podido encontrar tan profundamente en la termodinámica. Pero en el origen apela a modelos más altos. El buen sentido es esencialmente repartidor; su fórmula es "por una parte y por otra parte", pero la repartición que opera se hace en condiciones tales que la diferencia es puesta al comienzo, tomada en un movimiento dirigido que se supone colmarla, igualarla, anularla, compensarla (Deleuze, 1969: 93).

Deleuze establece allí una relación entre sentido y tiempo inspirada en la termodinámica. Si bien la discusión con la termodinámica está muy presente en el quinto capítulo de Diferencia y repetición, nos limitaremos aquí al punto principal. Un sistema termodinámico funciona por diferencias de temperatura que tienden naturalmente a equilibrarse al entrar en contacto. Manuel DeLanda lo ilustra con un ejemplo de gran claridad:

Si se crea un contenedor separado en dos compartimentos, y se llena uno de los compartimentos con aire frío y el otro con aire caliente, se crea con ello un sistema que encarna una diferencia en intensidad, siendo la intensidad en este caso la temperatura. Si se hace a continuación un pequeño agujero en la pared que divide los compartimentos, la diferencia intensiva causa el inicio de un flujo espontáneo de aire de un lado al otro (DeLanda, 1998).

Este es el principio de funcionamiento, por ejemplo, de una máquina a vapor, mediante la cual la energía térmica es transformada en energía mecánica. Ahora bien, según el segundo principio de la termodinámica, en cada uno de estos procesos, una parte de la energía se pierde en forma de calor en los alrededores del sistema, de tal modo que ya no es reutilizable. Así, la entropía o grado 
de equilibrio de un sistema va siempre en aumento, a la par de la degradación de la energía. Si la diferencia de temperatura inicial -diferencia de intensidad- es capaz de generar movimiento, el tiempo es el proceso mediante el cual la energía productiva se desvanece, es decir, el proceso mediante el cual se anula esa diferencia inicial. De allí que, según las leyes de la termodinámica, llegará un momento en el que ya no haya más energía utilizable, lo cual es denominado en este contexto "la muerte calórica del universo". Deleuze puede pensar entonces el buen sentido como anulación de la diferencia puesto que al repartir, equilibra las diferencias de intensidad, produciendo un estado de homogeneidad yerma. La interpretación ontológica que realiza Deleuze de la termodinámica (en tanto expresión de la concepción temporal correspondiente al buen sentido) es pues que la dirección impuesta al sentido por el buen sentido es la que tiende hacia lo indiferenciado, lo previsible, la anulación de la diferencia: la flecha del tiempo como escatología universal de la identidad en la indiferencia.

En Diferencia y repetición la cuestión está planteada en los mismos términos, con el agregado de que se establece una relación entre el buen sentido como modo de la temporalidad y uno de los principios genéticos de la temporalidad, conceptualizados por Deleuze como síntesis pasivas del tiempo:

El buen sentido se funda sobre una síntesis del tiempo, precisamente la que hemos determinado como la primera síntesis, la del hábito. El buen sentido no es bueno sino porque se ajusta al sentido del tiempo de acuerdo con esta síntesis. Testimoniando un presente viviente (y la fatiga de ese presente), va del pasado al futuro, como de lo particular a lo general. Pero define ese pasado como lo improbable o lo menos probable. En efecto, teniendo todo sistema parcial por origen una diferencia que individualiza su dominio, ¿Cómo un observador situado en el sistema aprehendería la diferencia de otro modo que como pasada, y altamente "improbable", 
puesto que está detrás de él? En contraste, en el seno del mismo sistema, la flecha del tiempo, es decir el buen sentido, identifica: el futuro, lo probable, la anulación de la diferencia. Esta condición funda la previsión misma (Deleuze, 1968: 290-291).

Es preciso subrayar, en aras de evitar interpretaciones precipitadas, que Deleuze no dice que el buen sentido se identifique con la primera síntesis del tiempo, sino que se funda sobre ella. Esta diferencia es fundamental, puesto que la síntesis del hábito es, bajo diferentes nombres y con distintas reformulaciones, uno de esos elementos que recorren toda la obra deleuziana y sin los cuales su filosofía no sería la que es, estando intrínsecamente relacionado con aquello a lo que se refiere a veces como "la vida inorgánica de las cosas" (Deleuze, 1985: 75; Deleuze/Guattari, 1991: 200). El buen sentido, por el contrario, no es un proceso de lo real mismo, sino una ilusión trascendental montada sobre lo real y pasible de ser combatida, al menos en cierta medida. Si el buen sentido puede montarse sobre la síntesis del hábito, sin embargo, es porque ésta implica una fatiga en cada contracción, fatiga que es reinterpretada por la termodinámica en términos de degradación energética. Para Deleuze, la síntesis del hábito es sólo un aspecto de la constitución y transformación de lo real, y de ahí que haya otros elementos en juego cuya productividad pueda compensar con creces las fatigas del hábito. Mientras que la primera síntesis del tiempo es necesaria, no debe ser tomada por la única modalidad de la temporalización.

El arma que utiliza Deleuze contra el poder del buen sentido es la paradoja. La paradoja del devenir que da comienzo a Lógica del sentido provoca ante todo una disrupción en la metafísica de la presencia propia del buen sentido, dado que un proceso de transformación no puede ser determinado en tiempo presente. Si decimos que Alicia crece, debemos decir que deviene más grande de lo que era y más chica de lo que es. No hay allí ningún contrasentido, pues lo que se dice no es que es al mismo tiempo más grande y más 
chica, sino que deviene al mismo tiempo más grande y más chica -de allí que la paradoja se llame "Del puro devenir" y no "Del puro ser"-. Enfocar el devenir en vez del ser otorga dos ventajas en lo que concierne a la temporalidad: no sólo nos fuerza a iluminar un modo del tiempo irreductible al instante o al "ahora", sino que también impide determinar una dirección única al sentido del tiempo. "El buen sentido es la afirmación de que, en todas las cosas, hay un sentido determinable; pero la paradoja es la afirmación de los dos sentidos a la vez" (Deleuze, 1969: 9). El acontecimiento destruye la flecha del tiempo, puesto que el devenir (crecer-empequeñecer) va en los dos sentidos a la vez. Pero como consecuencia de su ruptura con el buen sentido del tiempo, la paradoja provoca al mismo tiempo una ruptura con el sentido común correlativo, que ya no puede sostenerse en su tarea identitaria. "La paradoja es en primer lugar lo que destruye el buen sentido como sentido único, pero en segundo lugar lo que destruye el sentido común como asignación de identidades fijas" (Deleuze, 1969: 12).

La paradoja, entonces, rompe con el sentido común porque rompe en primera instancia con el buen sentido. Lo sublime, en contraste, rompe con el buen sentido porque rompe en primera instancia con el sentido común. Mientras que la paradoja es la herramienta privilegiada en Lógica del sentido, el encuentro sublime que violenta la sensibilidad e impide la concordia facultatum es la herramienta privilegiada en Diferencia y repetición. La paradoja "es el derrocamiento simultáneo del buen sentido y del sentido común: aparece por una parte como los dos sentidos a la vez de un devenir-loco, imprevisible; por otra parte, como el sinsentido de la identidad perdida, irreconocible" (Deleuze, 1969: 96). El término "simultáneo" no debe ser tomado allí en un sentido lógico: es focalizando el devenir-loco (es decir, rompiendo con el buen sentido) que la paradoja puede sustraerse a la identidad (es decir, romper con el sentido común). Es por ello que Deleuze puede 
preguntarse: “¿Cómo tendría Alicia aún un sentido común, no teniendo ya buen sentido?” (Deleuze, 1969: 97) En la irrupción de la intensidad en una experiencia sensible abrumadora el resultado es el mismo, pero el orden lógico del derrumbe es el inverso. Se trata de dos maneras distintas, aunque correlativas, de quebrar el mismo dispositivo, dependiendo de dónde se encuentre el eslabón débil de la cadena.

Antes de finalizar el apartado, respondamos a la siguiente pregunta: ¿por qué, en un libro intitulado Lógica del sentido, hay un capítulo o "serie" dedicado al sentido común y al buen sentido? Porque el sentido común y el buen sentido, vale recalcarlo, son modalidades del sentido. Esto implica decir que el sentido puede darse también de otras maneras: en efecto, "es lo propio del sentido no tener dirección, no tener 'buen sentido', sino siempre las dos [direcciones] a la vez, en un pasado-futuro infinitamente subdivido y prolongado" (Deleuze, 1969: 95).

A la línea orientada del presente, que "regulariza" en un sistema individual cada punto singular que recibe, se opone la línea del Aion, que salta de una singularidad pre-individual a otra y las retoma a todas, unas en las otras, retoma todos los sistemas siguiendo las figuras de la distribución nómade donde cada acontecimiento es ya pasado y aún futuro, más y menos a la vez, siempre la víspera y el día siguiente en la subdivisión que los hace comunicar conjuntamente (Deleuze, 1969: 95).

El esquema temporal triádico de Diferencia y repetición (triple síntesis del tiempo) se reduce en Lógica del sentido a un esquema dual: Chronos, el tiempo del presente de los cuerpos, se opone a Aion como tiempo de los acontecimientos incorporales; en este último, el presente ya no es localizable, dado el pasado-futuro simultáneo que implica el devenir. Este es el aspecto temporal de lo que De- 
leuze llama "repartición nómade"; en cuanto a su aspecto espacial, nos abocaremos a ello en el próximo apartado.

\section{El buen sentido como determinación espacial del sentido}

Le premier qui, ayant enclos un terrain, savisa de dire: Ceci est à moi, et trouva des gens assez simples pour le croire, fut le vrai fondateur de la société civile. Que de crimes, de guerres, de meurtres, que de misères et d'horreurs n'êेt point épargnés au genre humain celui qui, arrachant les pieux ou comblant le fossé, eût crié à ses semblables: Gardez-vous d'ecouter cet imposteur; vous êtes perdus, si vous oubliez que les fruits sont à tous, et que la terre n'est à personne.

RousseAu

El buen sentido no sólo impone una determinación temporal, sino también una determinación espacial al sentido. Como vimos, el buen sentido es esencialmente repartidor; sin embargo, su repartición puede caracterizarse aún más precisamente como una repartición sedentaria. "Una repartición tal implicada por el buen sentido se define precisamente como distribución fija o sedentaria" (Deleuze, 1969: 93). Deleuze relaciona una vez más el buen sentido con las clases medias pero también, lo que es aún más sugerente, con la cuestión agraria, la propiedad privada y la división de la sociedad en clases:

El buen sentido es agrícola, inseparable del problema agrario y de la instalación de cercados [enclos], inseparable de una operación de las clases medias en la que se supone que las partes se compensan, se regularizan. Máquina a vapor y ganadería en cercados, 
pero también propiedades y clases, son las fuentes vivas del buen sentido: no sólo como hechos que surgen en tal época, sino como eternos arquetipos; y no por simple metáfora, sino reuniendo todos los sentidos de los términos "propiedad" y "clases" (Deleuze, 1969: 94).

Del mismo modo, en Diferencia y repetición describe Deleuze lo que llama un nomos sedentario. Además de la relación entre el buen sentido y el sentido común con la repartición sedentaria, se ve allí una relación entre aquellos dispositivos y el juicio:

El sentido común y el buen sentido en tanto cualidades del juicio son entonces representadas como principios de repartición, que se declaran ellos mismos los mejores repartidos. Un tipo de distribución tal procede por determinaciones fijas y proporcionales, asimilables a "propiedades" o a territorios limitados en la representación. Puede ser que la cuestión agraria haya tenido una gran importancia en esta organización del juicio como facultad de distinguir partes ("por una parte y por otra parte"). Incluso entre los dioses, cada uno tiene su dominio, su categoría, sus atributos, y todos distribuyen a los mortales límites y lotes conformes a su destino (Deleuze, 1968: 54).

Se observa asimismo que el sentido común y el buen sentido son al mismo tiempo jueces y parte en tanto pretenden dar cuenta de sí mismos a partir de sí mismos. A este nomos sedentario, Deleuze contrapone un nomos nómade, "sin propiedad, cercado ni medida" (Deleuze, 1968: 54). ${ }^{10}$ En el último ya no hay reparto de lo

${ }^{10}$ Evidentemente, puede verse allí un antecedente del concepto deleuzo-guattariano de nomadismo (correlativo al de espacio liso), que los autores contraponen al de sedentarismo (correlativo al de espacio estriado). Dicho concepto no alude a una movilidad empírica, sino a una movilidad trascendental; como dicen Deleuze y Guattari, hay verdadero nomadismo incluso en "los que ya ni se mueven ni imitan nada", sino que "solamente agencian [agencent]" (De- 
distribuido, sino "repartición de aquellos que se distribuyen en un espacio abierto, ilimitado, al menos sin límites precisos" (Deleuze, 1968: 54). La instancia distribuidora y la instancia distribuida, a diferencia de lo que sucede en el nomos sedentario, coinciden. Si bien Deleuze continúa hablando de distribución, habría que decir en rigor que la tierra, más que distribuida, es poblada u ocupada.

Tengamos en cuenta que en este contexto Deleuze está discutiendo el problema de la univocidad del ser, con lo cual hay que entender estos dos tipos de distribución espacial no sólo en un sentido físico sino también, correlativamente, en un sentido ontológico. El nomos o la repartición sedentaria no sólo divide, cerca y reparte lotes en la tierra para la ganadería y la agricultura, sino que también separa y reparte lotes en el ser. Estos lotes son las categorías que, de Aristóteles a Kant, fragmentan el sentido del ser y producen en consecuencia una unidad tan sólo analógica del mismo. La instancia que se ocupa de ello, como queda claro en Kant, es el

leuze/Guattari, 1980: 35). "Así, es falso definir al nómade por el movimiento. Toynbee tiene mucha razón cuando sugiere que el nómade es sobre todo el que no se mueve" (Deleuze/Guattari, 1980: 472). El nomadismo es definido asimismo como velocidad o movimiento absoluto, del cual los viajes espirituales constituyen un ejemplo. "De este modo, hay que distinguir la velocidad y el movimiento: el movimiento puede ser muy rápido, pero no es por ello velocidad; la velocidad puede ser muy lenta, o incluso inmóvil, pero sigue siendo velocidad. El movimiento es extensivo, y la velocidad intensiva. El movimiento designa el carácter relativo de un cuerpo considerado como 'uno', y que va de un punto a otro; la velocidad, al contrario, constituye el carácter absoluto de un cuerpo cuyas partes irreductibles (átomos) ocupan o llenan un espacio liso a la manera de un torbellino, con la posibilidad de surgir en un punto cualquiera. (No es sorprendente, por lo tanto, que se haya podido invocar viajes espirituales que se hacían no en movimiento relativo, sino en intensidades en el lugar [sur place]: ellos forman parte del nomadismo). En suma, se dirá por convención que sólo el nómade tiene un movimiento absoluto, es decir, una velocidad; el movimiento turbulento [tourbillonnaire] o giratorio [tournant] que pertenece esencialmente a su máquina de guerra” (Deleuze/Guattari, 1980: 473). 
juicio. Hölderlin, en un brevísimo y genial opúsculo que extrañamente Deleuze nunca citó, "Urteil und Sein", lleva esa idea a su máxima potencia enfatizando el sentido etimológico de urteilen (juzgar) como un separar o repartir (teilen) originario (ur-), creando así el concepto de Urteilung como archi-división o repartición originaria (Cf. Hölderlin, 1962: 226-228). ${ }^{11}$

Por último, la cuestión de la distribución permite reforzar nuestra afirmación del apartado anterior respecto del estatuto ontológico derivado del buen sentido en contraposición a la producción de lo real. En efecto, Deleuze sostiene que el buen sentido juega un rol capital en la determinación del significado, pero ninguno en la determinación del sentido, "puesto que el buen sentido viene siempre en segundo lugar, porque la distribución sedentaria que opera presupone otra distribución, como el problema de los cercados supone un espacio en primer lugar libre, abierto, ilimitado" (Deleuze, 1969: 94). Puesto que el ser es pensado por Deleuze tanto en su temporalidad como en su espacialidad, señalemos respecto de la última que, si 1) la repartición sedentaria está asociada con la división en clases y el establecimiento de la propiedad privada, y a la vez 2) Deleuze deja en claro que este procedimiento se

\footnotetext{
${ }^{11}$ Amanda Núñez García sostiene que Hölderlin representa el germen del desvío schellinguiano respecto del idealismo, que abre "el horizonte idealista hacia la búsqueda de una exterioridad al Sistema", a "una fisura central en el mismo que le libere tanto de los fines, como de la estricta necesidad, como de la organicidad de este Absoluto cerrado y sin posibilidad de alteración" (Núnez García, 2010: 118). La razón de ello es que la crítica realizada por Hölderlin a Fichte en "Juicio y ser" con respecto a la dicotomía de la reflexión, que la distancia del Absoluto, habría conducido a tanto a Schelling como a Hegel a buscar una solución al problema. Sin embargo, mientras que la respuesta de Schelling vendría de la mano de una individuación que actúa por progresión, la respuesta hegeliana procedería por oposición. De este modo, la esencia se define en Schelling por la potencia, "más que por un esquema previo de la reflexión que debe doblarse en oposiciones desde el comienzo, aniquilando la simplicidad de lo Absoluto" (Núñez García, 2010: 116; cf., asimismo, Núñez García, 2012: 145-161).
} 
monta sobre un movimiento de territorialización previo, no resulta descabellado decir que el nomos nómade implica una suerte de "comunismo primitivo ontológico".

\section{Conclusiones}

En el transcurso de este trabajo hemos llegado a diferentes conclusiones. En primer lugar, identificamos tres sentidos en juego del término "sentido común": como el segundo postulado de la Imagen del pensamiento, relativo a la concordancia entre las facultades, como forma universal de identidad correlativa al buen sentido en tanto norma de repartición, y como imagen del pensamiento en tanto tal. En este último sentido, vimos que el sentido común implicaba suponer la buena voluntad del pensador y la naturaleza recta del pensamiento, supuestos que Deleuze contrapone a su empirismo trascendental en tanto exploración de un pensamiento no-dado de antemano. En cuanto al primer sentido, mostramos que el sentido común como concordia facultatum constituía un dispositivo que hacía converger los flujos de las facultades en los dos extremos: identidad de un objeto y de un sujeto, en contraposición a un ejercicio libre y discordante de las facultades no-sujeto a la condición de concordancia. Por último, en lo que concierne al sentido común como correlato del buen sentido, vimos que ambos elementos se complementaban conformando un mismo aparato, en el que el sentido común aporta la forma del objeto cualquiera y el buen sentido reparte y distribuye la experiencia en aras de cualificar dicho objeto.

Por otra parte, examinamos las determinaciones temporales y espaciales impuestas al sentido por el buen sentido. Mientras que el sentido sin más se ubica para Deleuze en una temporalidad del devenir irreductible al presente, el buen sentido impone una dirección única que va del pasado como lo más diferenciado hacia 
el futuro como indiferencia, constituyendo la flecha del tiempo en tanto escatología identitaria. Asimismo, mientras que el sentido efectúa una repartición nómade, el buen sentido opera una repartición sedentaria del espacio y del ser en lotes, fragmentando la univocidad del ser en la plurivocidad relativa de la analogía categorial. Esperamos con ello haber aportado elementos para una comprensión rigurosa de uno de los conceptos centrales de la filosofía deleuziana.

\section{Bibliografía}

De Landa, Manuel, 1998, "Deleuze and the Open-Ended Becoming of the World", en Chaos/Control: Complexity Conference, Universität Bielefeld. Disponible en: http://www.cddc.vt.edu/ host/delanda/pages/becoming.htm.

Deleuze, Gilles, 1963, La Philosophie critique de Kant, puf, París.

, 1968, Différence et répétition, PUF, París.

, 1969, Logique du sens, Minuit, París.

, 1985, Cinéma 2. L’Image-Temps, Minuit, París.

, 1990, Pourparlers. 1972-1990, Minuit, París.

, 1994, Difference and Repetition, Paul Patton (trad.), Athlone Press, Londres.

,2003, Deux régimes de fous. Textes et entretiens (1975-1995), David Lapoujade (ed.), Minuit, París.

, 2010, Derrames. Entre el capitalismo y la esquizofrenia, Equipo Editorial Cactus (trad.), Cactus, Buenos Aires.

, y Félix Guattari, 1980, Capitalisme et schizophrénie II. Mille plateaux, Minuit, París.

, y Félix Guattari, 1991, Qu'est-ce que la philosophie?, Minuit, París. 
París.

, y Claire Parnet, 1996 [1977], Dialogues, Flammarion,

Descartes, René, 1997, Meditaciones metafísicas y otros textos, E. López y M. Graña (trad.), Gredos, Madrid.

Dotti, Jorge Eugenio, 1986, "La distinción kantiana entre juicios de percepción y de experiencia”, Revista de Filosofía y Teoría Politica, núm. 26-27, La Plata, pp. 239-242.

Flaxman, Gregory, 2015, “A More Radical Empiricism”, en Deleuze and Pragmatism, Sean Bowden, Simone Bignall y Paul Patton, Routledge (comps.), Nueva York/ Londres, pp. 55-72.

Hegel, G.W.F., 1968, Gesammelte Werke. Band IV. Jenaer Kritische Schriften, Felix Meiner Verlag, Hamburgo.

, 1989, Diferencia entre el sistema de filosofía de Fichte y el de Schelling, Juan Antonio Rodríguez Tous (trad.), Alianza, Madrid.

,2012, La diferencia entre los sistemas filosóficos de Fichte y de Schelling, David Zapero Maier (trad.), Prometeo, Buenos Aires.

Heidegger, Martin, 2002, Was heisst denken (Gesamtausgabe. I. Abteilung: Veröffentlichte Schriften 1910-1976 — Band 8), Vittorio Klostermann, Frankfurt am Main.

Hölderlin, Friedrich, 1962, "Urteil und Sein", en Sämtliche Werke. 6 Bände, Band 4, F. Beissner, Stuttgart, pp. 226-228.

Kant, Immanuel, 1900 ss., Kants gesammelte Schriften. Abteilung 1: Werke: Band III: Kritik der reinen Vernunft (2. Aufl. 1787), Königlich Preußischen Akademie der Wissenschaften, Berlín.

, 2009, Crítica de la razón pura, Mario Caimi (trad.), Colihue, Buenos Aires.

Kerslake, Christian, 2009, Immanence and the Vertigo of Philosophy. From Kant to Deleuze, Edinburgh University Press, Edimburgo. 
Madelrieux, Stéphane, 2015, "Pluralism without Pragmatism: Deleuze and the Ambiguities of the French Reception of James", en Deleuze and Pragmatism, Sean Bowden, Simone Bignall y Paul Patton (comps.), Routledge, Nueva York/Londres, pp. 89-104.

Marx, Karl, 1987, Miseria de la filosofía. Respuesta a la Filosofía de la miseria de P.-J. Proudhon, Martí Soler (trad.), Siglo XxI, México.

Núñez García, Amanda, 2010, "La fisura del sistema: de Schelling y Hölderlin a Deleuze", en Actas del congreso "El fondo de la historia: idealismo, romanticismo y sus repercusiones", Madrid, pp. 114-121.

, 2012, "La grieta del sistema: Hölderlin entre Schelling y Deleuze", en Lógos. Anales del Seminario de Metafísica, vol. 45, Madrid, pp. 145-161.

Rölli, Marc, 2003, Gilles Deleuze. Philosophie des Transzendentalen Empirismus, Turia + Kant, Viena.

Sauvagnargues, Anne, 2009, Deleuze. L'empirisme transcendantal, PUF, París.

Wahl, Jean, 1953, Traité de métaphysique: Cours professés en Sorbonne, Payot, París. 